\title{
Plenary abstracts
}

PL.01

\section{Penicillin resistance in Streptococcus pneumoniae: evolution by genetic communication}

\author{
Regine Hakenbeck \\ Department of Microbiology, University of Kaiserslautern, Germany
}

In the early 80s it was discovered that penicillin-binding proteins (PBPs) are encoded by mosaic genes in penicillin resistant clinical isolates of Streptococcus pneumoniae as a result of gene transfer followed by recombination. It was soon recognised that commensal species are part of the game and might serve as potential donors for the mosaic sequences. In contrast, laboratory mutants resistant to beta-lactams contain point mutations in PBP genes, but also in other non-PBP genes. Where are we now? The astounding speed of rapidly evolving sequencing technologies has paved the way to extend our view from mosaic genes to interspecies gene transfer events affecting the entire genomes of the streptococcal world. I will also discuss molecular mechanisms contributing to beta-lactam resistance, based on the interplay of altered PBPs and other enzymes affecting the biosynthetic machinery of the bacterial cell wall.

PL.02

\section{Structure and assembly of the cell wall in Streptococcus pneumoniae}

\author{
Waldemar Vollmer \\ The Centre for Bacterial Cell Biology, Institute for Cell and Molecular Biosciences, Newcastle University, UK
}

The cell wall protects the bacterial cell from bursting due to its turgor, and maintains cell shape. The pneumococcal cell wall has a remarkably complex structure. The main component, peptidoglycan, carries several covalently attached macromolecules, including wall teichoic acid, capsular polysaccharide and cell surface proteins. Secondary structural modifications in the peptidoglycan, like $\mathrm{N}$-deacetylation and O-acetylation of the glycan chains, and the D-alanylation of teichoic acids contribute to the resistance of pneumococci against lysozyme and cationic antimicrobial peptides. Peptidoglycan and wall teichoic acid are also linked together by strong ionic interactions via divalent cations. So far we have gained only limited insights into the molecular mechanisms of pneumococcal cell wall synthesis, and the processes regulating cell wall synthesis during growth and division are largely unknown. Cell wall polymers are synthesised at the bacterial membrane and inserted into the existing wall. In the last steps phosphotransferases of the LytR-Cps2APsr family of enzymes (LCP enzymes) ligate the wall teichoic acid and capsular polysaccharides to the glycan chains of peptidoglycan to assemble the final cell wall structure. Cell wall growth is temporarily and spatially regulated to maintain the characteristic ovoid cell shape and to reliably divide the cell into two daughters. Lactic acid bacteria including pneumococci lack the cell elongation protein MreB which is present in many rod-shaped bacteria. The insertion of new material occurs at or near mid-cell where two modes of cell wall synthesis, one for elongation and another for cell division, take place simultaneously to produce the elongated, ovococcal cell shape.

\section{PL.03}

\section{Immuno-regulatory control of host susceptibility to pneumococcal carriage and invasive disease}

\author{
Aras Kadioglu \\ University of Liverpool, Liverpool, UK
}

Pneumococcal nasopharyngeal carriage is considered to be a prerequisite for invasive disease, but the majority of carriage episodes are asymptomatic and self-resolving. Interactions determining the development of carriage versus invasive 
disease, and in particular the immunological mechanisms underpinning nasopharyngeal carriage, are poorly understood but are crucial to deciphering why and how carriage may lead to invasive disease as well as the effectiveness of vaccines or therapeutics. I will describe the mechanisms by which Streptococcus pneumoniae colonise the nasopharynx without inducing damaging host inflammation and provide insight into the role of bacterial and host constituents that allow and maintain stable carriage, while also describing the factors whose perturbation lead to invasive disease. Similarly, I will discuss our current understanding of the role of immune regulation during invasive disease and how these factors contribute to host susceptibility. Overall, I will highlight the delicate balance between the requirements of the host to prevent inflammatory tissue damage with the need to combat potentially dangerous pathogen colonisation. This equilibrium needs to be considered carefully in the future design of anti-pneumococcal therapeutics and vaccines which may alter the density of pneumococcal carriage and hence affect upper airway immune responses.

PL.04

\title{
An ecological perspective on symbiosis between Streptococcus pneumoniae and the host
}

\author{
Debby Bogaert \\ UMCU-WKZ, The Netherlands
}

Respiratory tract infections are a major global health concern, accounting for high morbidity and mortality, especially in young children and elderly individuals. Traditionally, it is thought that bacterial respiratory tract infections, including otitis media and pneumonia, are caused by a limited number of pathogens like Streptococcus pneumoniae, and Haemophilus influenzae. The ecological niche for these potential pathogens is the upper respiratory tract of humans. They commonly reside here asymptomatically, and at equilibrium with their environment and the host. It is still unclear why in one individual an infection may develop, and in the other one not. However, over the last years the importance of the upper respiratory tract (URT) microbiota in maintaining respiratory health has become more apparent. Analogous to the gut microbiome, the respiratory microbiome is thought to be beneficial to the host by priming the immune system and providing colonisation resistance. In contrast, an imbalanced ecosystem lacking keystone bacteria might predispose to bacterial overgrowth, dissemination and consecutive respiratory infections. Recently, we have characterised the upper respiratory microbiota of a large group of individuals, young and old, during health and disease. We obtained evidence for the existence of different respiratory microbiota profiles, related to environmental drivers, to stability of the ecosystem and susceptibility to respiratory infections. Several of these profiles are positively or negatively related to streptococcal colonisation in general, and S. pneumoniae colonisation in particular. In this lecture I will present the current body of evidence regarding the position of $S$. pneumoniae within the nasopharyngeal and oropharyngeal microbial ecosystem, and discuss the current ecological theory regarding pathogenesis of respiratory infections.

\section{Poster abstracts}

\author{
P1.01
}

\section{Growth phase regulates the localisation and activity of the pneumococcal autolysin LytA}

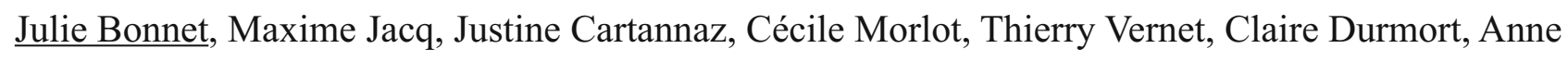
Marie Di Guilmi

Institut de Biologie Structurale, Grenoble, France

In Streptococcus pneumoniae, the choline-binding proteins (CBPs) are exposed at the cell surface through association to phosphocholine residues (PCho), which decorate the cell wall teichoic acids. Among them, LytA is an N-acetylmuramoylL-alanine amidase responsible for the pneumococcal autolysis in late stationary phase. Many aspects of LytA mechanism remain unknown, such as the molecular mechanism of release, the localisation pattern as well as the regulation of amidase activity. We have developed a version of the superfolder Green Fluorescent Protein (sfGFPop) appropriate to investigate the localisation of secreted proteins at the surface of $S$. pneumoniae. The fusion protein LytA-sfGFPop 